\title{
On the Link Between Insertion Loss and Lower Bound of Efficiency
}

\author{
Levon A. Barsikyan ${ }^{\# 1}$, Jason B. Coder ${ }^{* 2}$, Mark Gołkowski ${ }^{\# 3}$, John M. Ladbury *4 \\ \# Department of Electrical Engineering, University of Colorado Denver \\ 1205 5th St, Denver, CO 80204 \\ ${ }^{1}$ levon.barsikyan@ucdenver.edu \\ ${ }^{3}$ mark.golkowski@ucdenver.edu \\ * National Institute of Standards and Technology \\ 325 Broadway, Mail Stop 687.02, Boulder, CO 80305 \\ 2 jason.coderenist.gov \\ 4 john. ladburyanist.gov
}

\begin{abstract}
We investigate the effect of chamber loss on the recently developed 2-port antenna model and the associated method of determining antenna efficiency. The loss in the reverberation chamber was controlled using microwave absorbing foam creating four different configurations. For each configuration, the lower bound of transmitting efficiency of the antenna was calculated using the 2-port algorithm. The efficiency calculations are compared to the insertion loss in the reverberation chamber. The calculations show that as frequency increases, the calculated transmitting efficiency decreases in conjunction with the increased insertion loss. These results suggest the possibility of improving the lower bound calculation of the 2-port model in situations where chamber loss is known.
\end{abstract}

\section{INTRODUCTION}

The application and deployment of antennas continues to grow as the ability to transmit and receive information wirelessly is an integral part of products in everyday use. Antennas are generally classified under several parameters such as frequency, aperture, radiation, power handling capabilities and polarization. The radiating efficiency of an antenna is generally not a specified parameter, but one that can be useful to design engineers. Unfortunately, there are only limited number of empirical approaches to finding antenna efficiency, and are typically very time consuming and yield results with high uncertainties.

A recently developed 2-port network antenna model provides for novel empirical methods for characterizing antenna transmission and reception performance. This model also provides equations for finding the transmitting and receiving efficiencies from reverberation chamber measurements [1-4]. However, one shortfall of the 2-port network model algorithm is that it does not consider loss in the reverberation chamber. In fact, the model assumes that the chamber has negligible loss for at least a subset of measurements.

The goal of this paper is to explore the link between the insertion loss of the reverberation chamber and the calculated lower bound of transmitting efficiency. Ideally, it is desirable to quantify the insertion loss of the reverberation chamber and

U.S. Government work not protected by U.S. copyright correct the results of the 2-port model using that information. In Section II we outline the basics of the 2-port model and fundamental equations that have been derived to calculate the lower bound of transmitting and receiving efficiency along with insertion loss. After the model is defined, we show measured data for the lower bound of transmitting efficiency, insertion loss, and investigate the link between the two quantities.

\section{Description of the Two-Port Antenna Model}

The 2-port antenna model can be described as a method of modeling a real antenna as a perfectly matched and lossless antenna connected in series with a 2-port network [1-4]. This passive 2-port network contains all the imperfections of the antenna. Figure 1 shows a signal flow graph of the two port model along with the notation for the reflection and transmission parameters. This 2-port network is defined as having the same intrinsic impedance (in most cases $50 \mathrm{Ohms}$ ) at both the input and output ports [1-2]. The network is also reciprocal, that is: $T_{n}=R_{n}$ [1-2]. Although full characterization of the power flow through an antenna is achievable with the parameters of the 2-port network, the model does not encompass the geometry of the radiation pattern and scattering characteristics [1-4].

One common application of the 2-port model is determining the lower-bound of antenna efficiency. In [2-3], methods for determining the lower bound of radiation and receiving efficiencies are defined. These methods adhere to the definitions of antenna terms found in the IEEE 145 standard [5], but here, we ignore receiving efficiency because there is no established method to empirically measure it. In IEEE 145, radiation efficiency is defined as the ratio of power radiated by the antenna to the power accepted by the antenna [5]. From network theory, the radiation efficiency of the two-port network can be written as:

$$
\eta_{T}=\frac{\left|T_{n}\right|^{2}}{1-\left|\Gamma_{n}\right|^{2}},
$$

where $\eta_{T}$ denotes the radiation efficiency. In [2], the basic calculation of radiation efficiency, beginning with measured 


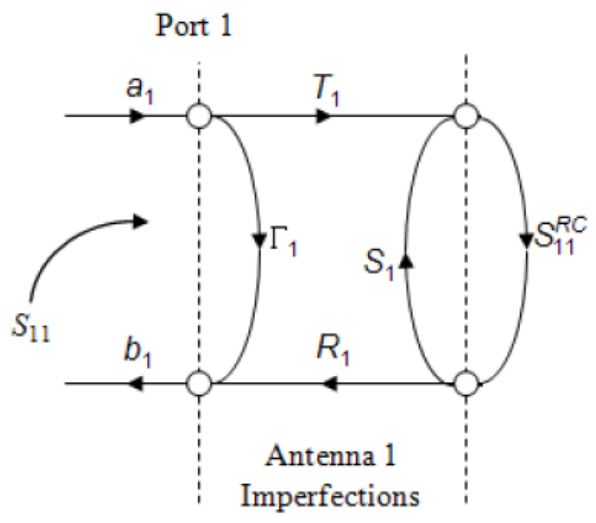

Fig. 1. Flow chart of a two-port network antenna model

data is described in detail. An improved method is presented in [4] and is designed to perform better in situations where the antenna is poorly matched. Both the original and revised method operate on the same key assumption: the transmitted signal is perfectly reflected back to the antenna for at least a few stirrer positions. From this assumption, it is evident that if the loss in the reverberation chamber is high, there will be no stirrer positions in which the signal is perfectly reflected back to the antenna. This will make the lower bound of radiation efficiency artificially low. In the extreme case, where the losses are very high (e.g. at high frequencies), the lower bound may be so low that it contains very little useful information. If we know loss in the reverberation chamber can significantly impact the calculation of the lower bound of radiation efficiency, can the effect be reversed? Here we investigate the relationship between insertion loss and the lower bound of radiation efficiency empirically.

\section{CAlculation of Insertion Loss}

To explore the relationship between the insertion loss and the lower bound of radiating efficiency, we first need to calculate the insertion loss. The established method of calculating insertion loss, $I L$, involves measuring the transmission $\left(S_{21}\right)$ from one antenna to another and correcting for the impedance mismatch of both antennas:

$$
I L=\frac{<\left|S_{21}\right|^{2}>}{\left(1-\left|<S_{11}>\right|^{2}\right)\left(1-\left|<S_{22}>\right|^{2}\right)},
$$

where all quantities are in terms of observed $S$-parameters, and not in terms of the model two-port network [6]. The above equation requires two antennas to be present in the reverberation chamber. Ideally, when utilizing the 2-port model, there should only be one antenna inside the reverberation chamber since the additional antenna becomes an unaccounted source of loss. To estimate insertion loss using the 2-port model the following equation can be used:

$$
I L=\frac{1}{2}<\left|S_{11}^{R C}\right|^{2}>
$$

Similar to (2) this equation is based on formulations in [6], but accomplishes the same as equation (2) with only a single antenna inside the reverberation chamber. The $1 / 2$ term on the left originates from the enhanced backscatter effect that is observed in the reverberation chamber [7]. One might expect to see a term in the numerator or denominator that corrects for the mismatch of the antenna being used to measure the insertion loss. In this case, such terms are not required because the mismtach of the measurement antenna is already accounted for in the solution of $S_{11}^{R C}$. The solution for $S_{11}^{R C}$ is found in [4] as:

$$
S_{11}^{R C}=\frac{S_{11}-\Gamma_{1}}{T_{1} R_{1}+\left(S_{11}-\Gamma_{1}\right) S_{1}} .
$$

$S_{11}$ is directly measured using a vector network analyzer (VNA). From [4], we also know that the ensemble average (denoted as $<>$ ) of $S_{11}$ is equal to $\Gamma_{1}$. The $T, R$, and $S$ parameters are then calculated assuming the antenna inside the reverberation chamber is $100 \%$ efficient. The assumption that the antenna is $100 \%$ efficient is used as a starting point. In reality, we don't know the efficiency of the antenna, but in this case we are using a well constructed monopole as described in Section IV. Because of this assumption, this calculation of insertion loss is strictly an estimation.

\section{Measurements}

To test the hypothesis that the insertion loss directly influences the lower bound of radiation efficiency, we placed two virtually identical monopoles inside the working volume of a reverberation chamber and measured all four complex $S$-parameters with a VNA. Although the presence of two antennas inside the reverberation chamber creates an extra loss, the effect should be minimal.

The chamber's dimensions consist of $4.2 \mathrm{~m}$ in length, $3.6 \mathrm{~m}$ in width, and $2.9 \mathrm{~m}$ in height. Inside the chamber two shifting stirrers are mounted from wall to wall: one placed vertical and the other horizontal. Both of the paddles are the same by design and measure $0.7 \mathrm{~m}$ wide.

The antennas under test (AUTs) were monopoles with a circular ground plane measuring at a diameter of $298.4 \mathrm{~mm}$. The diameter of the monopole measured at $6.4 \mathrm{~mm}$ with a length of $100.4 \mathrm{~mm}$. Complex $S$-parameter data were acquired with a VNA over 529 stepped stirrer positions.

The monopole antennas were designed and constructed to facilitate easy theoretical modeling and analysis. For a monopole, Ohmic losses can be calculated analytically from the known current distribution and material conductivity. This theoretical analysis yields an idea of how close the lower bound of radiation efficiency is to the true radiation efficiency.

Figure 2 displays the theoretical transmission efficiency of this monopole as well as the lower bound on the transmitting efficiency calculated using the 2-port antenna model for the case when the chamber is unloaded. The theoretical transmission efficiency is very close to unity over the entire frequency range due to the high conductivity of copper, and the fact that the definition of efficiency used is independent of mismatch. 


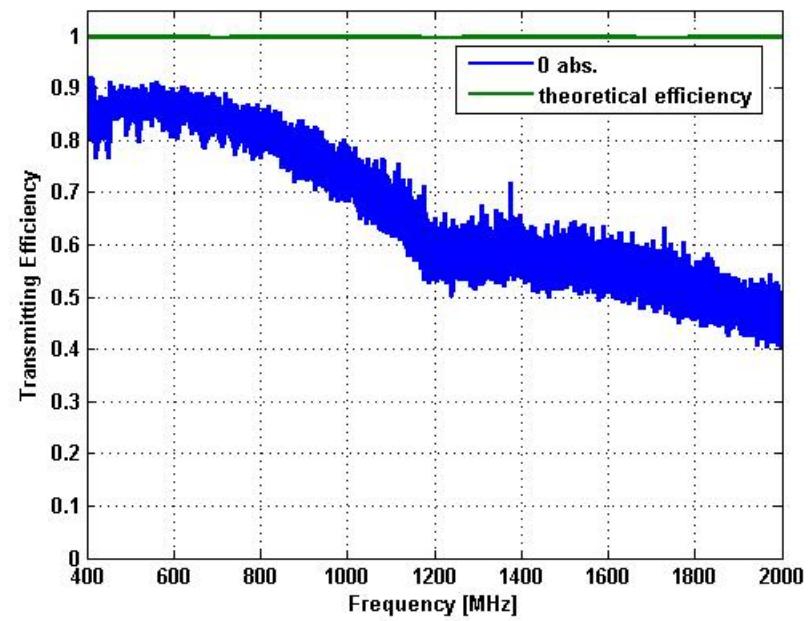

Fig. 2. Theoretical efficiency and measured lower bound transmitting efficiency for zero microwave absorbers as a function of frequency. Theoretical efficiency is near unity over the frequency range, while calculated transmitting efficiency falls off with increasing frequency. The cause of the inflection point at $1200 \mathrm{MHz}$ in the lower bound of transmitting efficiency is unknown, but is thought to result from interaction between the antenna and the environment.

At the same time, the lower bound calculated from the 2-port model significantly drops with increasing frequency. These results show that this departure of the 2-port model derived efficiency estimate from the theoretically expected value is due to frequency dependent chamber losses and violation of the assumption that the chamber yields a lossless reflection for at least one stirrer position.

In Figure 2, at approximately $1200 \mathrm{MHz}$ there is a point of inflection in the lower bound of transmitting efficiency. The exact cause of this is unknown, but the data have been examined and it appears the inflection is caused by an interaction between the antenna and the environment. It is not a characteristic of the AUT. Additional measurements to determine if the effect is dependent on the antennas position in the reverberation chamber would be required to pinpoint the exact cause.

With the lower bound and theoretical radiating efficiencies established, we can begin to introduce additional loss into the reverberation chamber. This additional loss was accomplished by adding 1-4 pieces of pyramidal absorbing foam.

Figure 3 shows the insertion loss of the reverberation chamber as calculated using (3) and (4). As expected, insertion loss noticeably increases as additional absorbing blocks are added to the reverberation chamber. The lower bound of radiation efficiency is also calculated using the same set of data, and is shown in Figure 4.

Figure 3 shows that with more absorbers in the system, insertion loss gets progressively higher, while the observations of Figure 4 suggest that the transmitting efficiency is decreasing with higher frequency. This should not be the case if the reverberation chamber is perfectly reflective for at least a few stirrer positions. These results indicate that the fall of of the

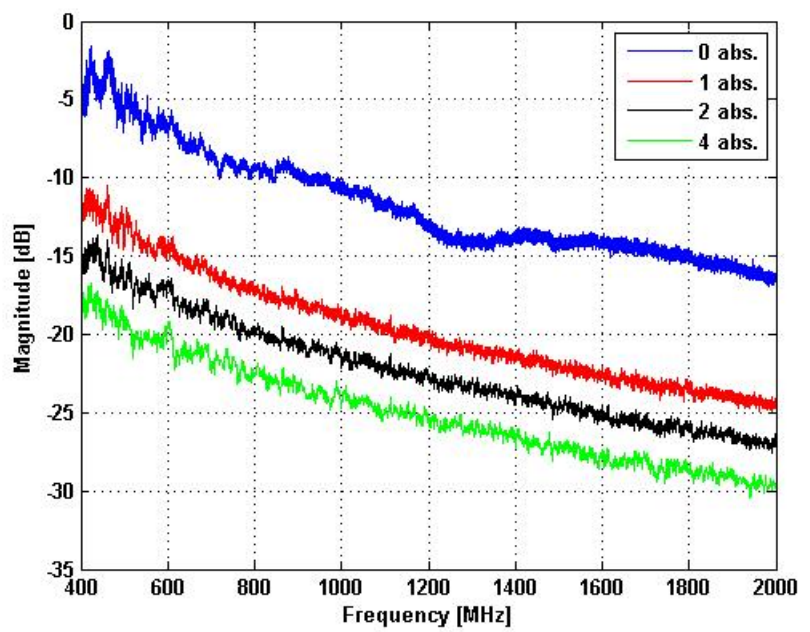

Fig. 3. Insertion loss calculations for four different absorber configurations as a function of frequency. As expected, insertion loss increases with a greater number of absorbers in the chamber.



Fig. 4. Lower bound transmitting efficiency calculations for each absorber configuration. Efficiency progressively decreases with a greater number of absorbers in the chamber. The cause of the inflection point at $1200 \mathrm{MHz}$ in the lower bound of transmitting efficiency is unknown, but is thought to result from interaction between the antenna and the environment inside the reverberation chamber.

radiation efficiency is due to larger chamber losses at higher frequencies.

\section{Discussion}

Evidence from our calculated data suggests that insertion loss dominates antenna efficiency calculations at higher frequencies. Figure 2 demonstrates that at lower frequencies the transmitting efficiency of the antenna calculated from measurements is close to the theoretical expected value. As the frequency increases efficiency falls off and insertion loss progressively increases. 


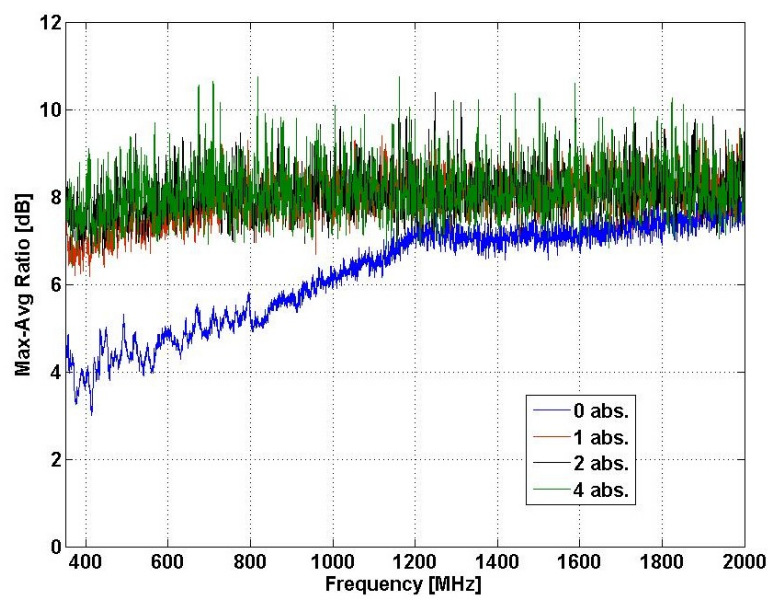

Fig. 5. Max-average ratio for each absorber configuration. An 11 point moving average has been applied to the max-average ratio data shown. Data are calculated after converting to $S_{11}^{R C}$.

When comparing Figure 3 and Figure 4 there is a distinctive commonality in the slopes, suggesting that there is a correlation between insertion loss and transmitting efficiency. If Figure 2 is an insight into the behavior of the reverberation chamber then we may be able to accurately include insertion loss in the 2-port antenna model to get a better efficiency calculation.

It must be noted that Figure 3 and Figure 4 cannot be directly compared because insertion loss is on a scale of $\mathrm{dB}$ and transmitting efficiency is in units of percentage. Thus the similarity in the slope is not a definitive indication of a causal relationship between the decrease in radiation efficiency and the insertion loss.

To better understand if there is a causal relationship between the lower bound of radiation efficiency and the insertion loss, we can examine the max-average ratio of the insertion loss data. This max-average ratio is calculated from the $S_{11}^{R C}$ data, before insertion loss is calculated. We expect to see an exponential distribution (approximately) of the squared magnitude, yielding a max-average ratio of 6.8 , or about 8 dB. Figure 5 shows the max-average ratio for each absorber configuration.

At higher frequencies, the max-average ratio of the $S_{11}^{R C}$ data are very close to the expected value of $8 \mathrm{~dB}$. This indicates that the distribution of the magnitudes is the same and is dominated by losses in the chamber. These losses create a more mathematically ideal exponential distribution with a very long tail. At lower frequencies and lower losses, we effectively see a truncated exponential distribution (where the upper limit is unity). The departure of the max-average ratio from $8 \mathrm{~dB}$ is clearly visible for frequencies below $1 \mathrm{GHz}$ for the case of 0 absorbers. This is a result of there being large reflections and indicates that the two-port model (and thus the calculation of efficiency) are more relevant and applicable. Because the 2-port model relies heavily on the distribution of the magnitude - the lower bound of efficiency is based on the radius of the smallest circle that bounds all of the data - a truncated distribution indicates the stirrer is nearly perfectly reflecting for at least a few positions.

A focus for future research could be to utilize the difference between the measured data and the analytic calculation. This difference may yield some information about the environment the antenna is operating in. Consider the case where an antenna is characterized in an ideal reverberation chamber. In this environment the two-port model is solved, and the parameters of the antenna become known. If the same antenna is then put in an unknown environment, any difference between the expected efficiency and the lower bound could be considered an aspect of the environment.

\section{CONCLUSION}

Using the 2-port network model, measurements were made to examine the link between the lower bound of radiation efficiency and the insertion loss of the reverberation chamber. Insertion loss was calculated by use of a modified equation that allows for only a single antenna to be present inside the reverberation chamber. Comparison of transmitting efficiency values and insertion loss values yields similar trends. The results show that with increasing frequency the calculated relative efficiency decreases and the insertion loss increases. For future work, these results suggest the possibility of correcting and improving the radiation efficiency calculation in situations where chamber loss is known.

\section{REFERENCES}

[1] J. M. Ladbury and D. A. Hill, "An Improved Model for Antennas in Reverberation Chamber," IEEE International Symposium on EMC. July 2010.

[2] J. B. Coder, J. M. Ladbury, and M. Gołkowski, ”A Novel Method for Determining the Lower Bound of Antenna Efficiency," IEEE International Symposium on EMC. August 2011.

[3] J. B. Coder, J. M. Ladbury, and M. Gołkowski, "Two Port Model for Antennas in an Arbitrary Environment," IEEE Transactions on Antennas and Propagation, manuscript AP1211-1524, publication pending.

[4] J. B. Coder, J. M. Ladbury and M. Gołkowski, "On Lower Bound Antenna Efficiency Measurements in a Reverberation Chamber," IEEE International Symposium on EMC. August 2012.

[5] IEEE Antenna Standards Committee, "IEEE Standard Definitions of Terms for Antennas," March, 1993.

[6] J. M. Ladbury, G. H. Koepke, and D. G. Camell, "Evaluation of the NASA Langley Research Center Mode-Stirred Chamber Facility," National Institute of Standards and Technology Technical Note 1508 January 1999.

[7] J. M. Ladbury and D. A. Hill "Enhanced Backscatter in a Reverberation Chamber" IEEE International Symposium on EMC July 2007. 